\title{
Strong Orbital Fluctuations in Multipolar Ordered States of $\operatorname{PrV}_{2} \mathrm{Al}_{20}$
}

\author{
Yosuke Matsumoto*, Masaki Tsujimoto, Takahiro Tomita, Akito Sakai and Satoru Nakatsuji \\ Institute for Solid State Physics, University of Tokyo, Kashiwa, Japan
}

\begin{abstract}
$\operatorname{Pr}_{2} \mathrm{Al}_{20}(T=\mathrm{Ti}, \mathrm{V})$ are ideal systems to study the quadrupole Kondo effect and quantum criticality arising from orbital degrees of freedom. The both systems have the nonmagnetic cubic $\Gamma_{3}$ crystal electric field ground doublet with the well separated excited state. In particular, $\operatorname{PrV}_{2} \mathrm{Al}_{20}$ exhibits anomalous metallic behavior above and below the multipolar ordering temperatures, reflecting the even stronger hybridization between $f$ and conduction electrons possibly due to a proximity to a orbital quantum critical point. Here we report the anomalous metallic behaviors found in a pure single crystal of $\operatorname{PrV}_{2} \mathrm{Al}_{20}$. Our detailed analyses revealed that the resistivity indicates power law temperature dependence proportional to $T^{3}$. Furthermore, we pointed out that the $4 f$ electron contribution to the specific heat also exhibits power law behavior proportional to $T^{4}$. Both observations are in a sharp contrast to the gapped behavior found in $\operatorname{PrTi}_{2} \mathrm{Al}_{20}$ and indicate the strong $c$ - $f$ hybridization and strong orbital fluctuations in $\operatorname{PrV}_{2} \mathrm{Al}_{20}$. In addition, the $4 f$ electron contribution to the entropy in $\mathrm{PrV}_{2} \mathrm{Al}_{20}$ reaches only $50 \%$ of $R \ln 2$ at an orbital ordering at $T=0.75$ $\mathrm{K}$, suggesting another $50 \%$ of $R \ln 2$ expected for $\Gamma_{3}$ doublet is already released at higher temperature possibly due to quadrupole Kondo effect.
\end{abstract}

Keywords: orbital fluctuations, multipole ordering, quadrupole Kondo effect, unconventional superconductivity

\section{Introduction}

Novel quantum phases may arise from $f$ electrons' orbital degrees of freedom through hybridization with conduction electrons. For instance, quadrupole Kondo effect, originally suggested as a single ion effect in the nonmagnetic cubic $\Gamma_{3}$ crystal electric field (CEF) doublet[1], has attracted a lot of attention because of its non-Fermi liquid ground state. It is an interesting open question what happens in the lattice systems. On the other hand, it is a further non-trivial open issue to search for a novel quantum phase arises in the vicinity of a quantum critical point (QCP) of an orbital ordering. So far, a lot of interesting quantum states, such as exotic superconductivity (SC) with highly enhanced effective mass and non-Fermi liquid, have been reported in the vicinity of magnetic QCPs[2, 3, 4]. Similarly exotic states may also be found at the border of orbital QCPs.

In order to study this, it is important to chose a material which has purely orbital degrees of freedom in the

\footnotetext{
${ }^{*}$ Corresponding author

Email address: matsumoto@issp.u-tokyo.ac.jp (Yosuke Matsumoto)
}

Preprint submitted to Nuclear Physics B ground state. Such examples can be found in cubic $\Gamma_{3}$ doublet systems of a $f^{2}$ configuration. Intensive studies have revealed various interesting phenomena in the cubic $\Gamma_{3}$ doublet systems, in particular, in Pr-based intermetallic compounds[5, 6, 7, 8, 9]. However, Pr-based cubic $\Gamma_{3}$ doublet systems usually have well-localized $f$ moments, and thus there has been no prototypical system where we can study the orbital instability by tuning the hybridization between $f$ and conduction electrons.

On the other hand, recent studies have revealed that $\operatorname{Pr} T_{2} \mathrm{Al}_{20}(T=\mathrm{Ti}, \mathrm{V})$ are ideal systems to study the quadrupole Kondo effect and quantum criticality arising from orbital degrees of freedom, where we can tune the $c$ - $f$ hybridization by chemical substitution and by pressure[10,11]. The both systems have the nonmagnetic cubic $\Gamma_{3}$ CEF doublet with the well separated excited state at $\Delta_{\mathrm{CEF}} \sim 60 \mathrm{~K}(\mathrm{Ti})$ and $40 \mathrm{~K}(\mathrm{~V})[10,12,13]$. In addition, strong hybridization is expected in these compounds, from a caged structure with $16 \mathrm{Al}$ ions surrounding $T$ ions. Indeed, the hybridization is strong as is evident in many physical properties[10, 14, 15, 16]. In particular, $\operatorname{PrV}_{2} \mathrm{Al}_{20}$ exhibits anomalous metallic behavior above and below the multipolar ordering temperatures, reflecting the even stronger hybridization due to 
a smaller size of the cage.

$\mathrm{PrV}_{2} \mathrm{Al}_{20}$ exhibits double multipolar orderings at temperatures $T=0.65$ and $0.75 \mathrm{~K}$, which has been revealed by our recent specific heat measurements using a pure single crystal $[17,16]$. The double transition becomes more prominent in the samples with better quality, which indicates that the double transition is the intrinsic property of $\operatorname{PrV}_{2} \mathrm{Al}_{20}$. However, the origin of the double transition is not clear so far. On the other hand, a ferro-quadrupole (FQ) ordering (at $T=2.0 \mathrm{~K}$ ) has been suggested for $\operatorname{PrTi}_{2} \mathrm{Al}_{20}[10,12,18]$. Interestingly, both of these compounds exhibit heavy fermion SC inside the mutipole ordering phases[19, 11, 16]. In $\operatorname{PrTi}_{2} \mathrm{Al}_{20}$, the $\mathrm{SC}$ is highly enhanced under pressure of $8 \mathrm{GPa}$, where the ferro-quadrupole ordering starts to be suppressed. The transition temperature $\left(T_{\mathrm{c}}\right)$ at ambient pressure $(\sim 0.2 \mathrm{~K})$ increases up to $\sim 1 \mathrm{~K}$ and the effective mass reaches $m^{*} / m_{0} \sim 110[11]$. This strongly suggests a novel heavy fermion $\mathrm{SC}$ emerges in the vicinity of the orbital QCP. Moreover, in the case of $\mathrm{PrV}_{2} \mathrm{Al}_{20}$, the SC appears at $T_{\mathrm{c}}=0.05 \mathrm{~K}$ with the highly enhanced effective mass $\left(m^{*} / m_{0} \sim 140\right)$ even at ambient pressure[16]. This observation indicates the first realization of the novel SC arising from the orbital fluctuation of the $f$ electrons at ambient pressure.

Here, we discuss the anomalous metallic behaviors found below and above the multipole ordering temperatures in a pure single crystal of $\operatorname{PrV}_{2} \mathrm{Al}_{20}[16,20]$, focusing on how they are different from those found in $\operatorname{PrTi}_{2} \mathrm{Al}_{20}$. We revealed that the resistivity of $\operatorname{PrV}_{2} \mathrm{Al}_{20}$ indicates power law temperature dependence proportional to $T^{3}$, which is in contrast to the gapped behavior found in $\operatorname{PrTi}_{2} \mathrm{Al}_{20}$. Likewise, the $4 f$ electron contribution to the specific heat of $\operatorname{PrV}_{2} \mathrm{Al}_{20}$ exhibits power law behavior proportional to $T^{4}$, which is again in contrast to the gapped behavior in $\operatorname{PrTi}_{2} \mathrm{Al}_{20}$. In addition, the $4 f$ electron contribution to the entropy in $\operatorname{PrV}_{2} \mathrm{Al}_{20}$ reaches only $50 \%$ of $R \ln 2$ at the orbital ordering at $T=0.75 \mathrm{~K}$, suggesting another $50 \%$ of $R \ln 2$ expected for $\Gamma_{3}$ doublet is already released at higher temperature possibly due to quadrupole Kondo effect. All these observations indicate the strong $c$ - $f$ hybridization and strong orbital fluctuations which may arise from the proximity to a orbital QCP in $\operatorname{PrV}_{2} \mathrm{Al}_{20}$. Note that a part of the results presented in this paper has been already discussed in the previous works[10, 16, 20].

\section{Experimental}

Single crystals of $R T_{2} \mathrm{Al}_{20}(R=\mathrm{Pr}, \mathrm{La}, T=\mathrm{Ti}$, $\mathrm{V})$ were grown by the Al self-flux method[10]. We have succeeded in growing high quality single crystals, whose residual resistivity ratio (RRR) reaches $\sim 20$, by tuning the starting ratio. Single and powder X-ray diffraction and SEM-EDX measurements indicate high quality and single phase of obtained crystals. The electrical resistivity $\rho(T)$ was measured by a low-frequency ac four-terminal method below $\sim 1 \mathrm{~K}$ using ${ }^{3} \mathrm{He}-{ }^{4} \mathrm{He}$ dilution refrigerator. A dc four-terminal method was used above $T=0.3 \mathrm{~K}$ in conjunction with a commercial system (PPMS, Quantum Design). In this paper, RRR is roughly defined as the ratio between the values at $300 \mathrm{~K}$ and lowest temperature above $T_{\mathrm{c}}$ of the SC transition. The specific heat was measured using a relaxation method for the pure single crystal with RRR $\sim 20(0.111 \mathrm{mg})$, which is the same sample as used for the ac- and dc-susceptibility measurements in the previous studies[16]. For the measurements above $T=0.9$ $\mathrm{K}$, a commercial system (PPMS, Quantum Design) was used. Measurements below $T \sim 1 \mathrm{~K}$ were made by using a specific heat cell installed in a ${ }^{3} \mathrm{He}-{ }^{4} \mathrm{He}$ dilution refrigerator. The details were already discussed in the previous work[16].

\section{Results and Discussions}

First we present the low temperature resistivity $\rho$ for various single crystals of $\operatorname{PrV}_{2} \mathrm{Al}_{20}$ with different sample qualities in Figure 1 (a). Here, the data for the single crystal of $\operatorname{PrTi}_{2} \mathrm{Al}_{20}(\mathrm{RRR} \sim 150)$ are also shown for comparison. As clearly seen in the figure, $\operatorname{PrV}_{2} \mathrm{Al}_{20}$ with $\mathrm{RRR} \sim 19$ exhibits a resistivity drop due to the SC transition at $T_{\mathrm{c}}=0.05 \mathrm{~K}$, as indicated by an arrow. The $\mathrm{SC}$ transition was also found in another sample (RRR $\sim 18$ ) at a slightly lower $T_{\mathrm{c}}=0.039 \mathrm{~K}$. However, we did not find any sign of SC at least down to 38 and 43 $\mathrm{mK}$ for the sample with RRR $\sim 4$ and 7 , respectively. The results suggest that $T_{\mathrm{c}}$ strongly depends on RRR, which may indicate an unconventional character of the SC. Apparently, further measurements are required to confirm the RRR dependence.

On the other hand, there is another relatively steep drop of the resistivity at $T_{\mathrm{O}} \sim 0.7 \mathrm{~K}$, which is associated with multipolar orderings of $\operatorname{PrV}_{2} \mathrm{Al}_{20}$. Here, we defined $T_{\mathrm{O}}$ as a peak temperature of $d \rho / d T$, which is dependent on the RRR of the sample[16]. While clear double transitions were found in the specific heat at $T_{\mathrm{Q}}=0.75 \mathrm{~K}$ and $T^{*}=0.65 \mathrm{~K}, d \rho / d T$ indicates only one peak even for the sample with RRR $\sim 19$ at least in zero-magnetic field. $T_{\mathrm{O}}$ increases from 0.5 to $0.67 \mathrm{~K}$ if the RRR is increased from $\sim 4$ to $\sim 19$. In the case of $\mathrm{PrTi}_{2} \mathrm{Al}_{20}, T_{\mathrm{O}}$ is found at $\sim 2.0 \mathrm{~K}$, which is associated with the FQ ordering. 

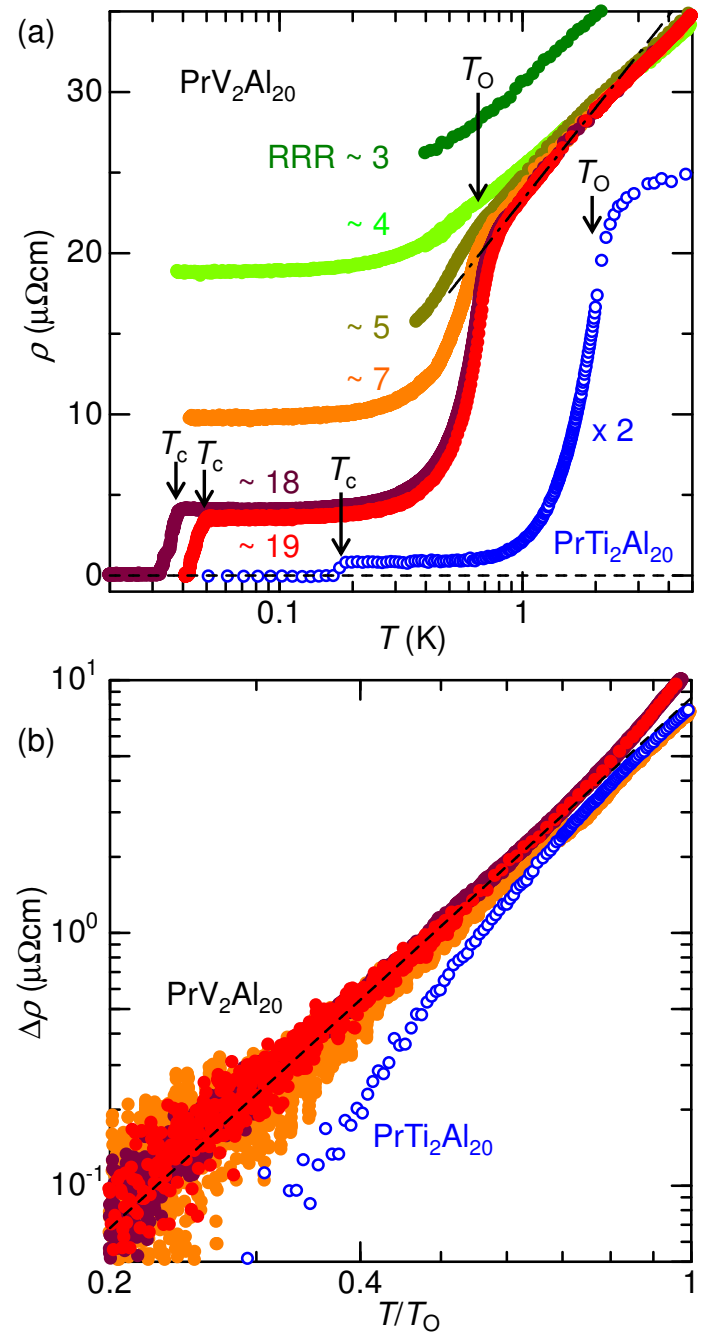

Figure 1: (a) Temperature dependence of the resistivity $\rho$ for various single crystals of $\mathrm{PrV}_{2} \mathrm{Al}_{20}$ with different sample qualities (filled circles). The data for the single crystal of $\operatorname{PrTi}_{2} \mathrm{Al}_{20}$ (RRR 150) are also shown for comparison (open circles). Note that the data for $\mathrm{PrTi}_{2} \mathrm{Al}_{20}$ are multiplied by a factor of two for clarity. The dashed line represents a fit by a $\ln T$ curve at $T>T_{\mathrm{O}}$ to the data for RRR $\sim 19$. (b) Full logarithmic plot of $\Delta \rho$ against normalized temperature $T / T_{\mathrm{O}}$ for $\operatorname{PrV}_{2} \mathrm{Al}_{20}$ and $\operatorname{PrTi}_{2} \mathrm{Al}_{20}$. Here, $\Delta \rho$ is defined as $\Delta \rho \equiv \rho-\rho_{0}$, where $\rho_{0}$ is the residual resistivity estimated above $T_{\mathrm{c}}$. The broken line indicate a power law temperature dependence of $\Delta \rho \propto T^{3}$. Note that the same symbols as (a) are used here. For details, see text.

In order to discuss the temperature dependence of the resistivity below $T_{\mathrm{O}}$ more in detail, we present a full logarithmic plot of $\Delta \rho$ against normalized temperature $T / T_{\mathrm{O}}$ for $\operatorname{PrV}_{2} \mathrm{Al}_{20}$ and $\operatorname{PrTi}_{2} \mathrm{Al}_{20}$ in Figure 1 (b). Here, $\Delta \rho$ is defined as $\Delta \rho \equiv \rho-\rho_{0}$, where $\rho_{0}$ is the residual resistivity estimated above $T_{\mathrm{c}}$. Remarkably, all the data collapse on top for $\operatorname{PrV}_{2} \mathrm{Al}_{20}$ with $\mathrm{RRR} \sim 7,18$

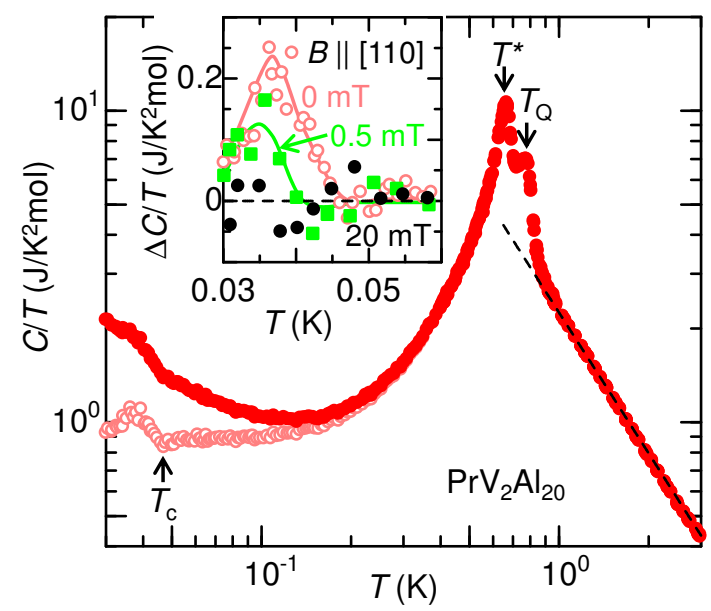

Figure 2: Temperature dependence of $C / T$ (closed circles). Here, the lattice contribution is subtracted by using $C / T$ for $\mathrm{LaV}_{2} \mathrm{Al}_{20} . C_{4 f} / T$ obtained after subtracting the nuclear contribution $C_{n} / T$ is also shown (open circles). The broken line at $T>T_{\mathrm{Q}}$ indicates the anomalous temperature dependence with $C / T \propto T^{-3 / 2}$. Inset shows $\Delta C / T$ obtained after subtracting $\gamma$ from $C_{4 f} / T$ at the lowest temperatures around $T_{\mathrm{c}}$. In addition to the zero-field data, those in the weak magnetic field along the [110] direction are also shown. See text for details.

and 19. Furthermore, these exhibit a power law temperature dependence of $\Delta \rho \propto T^{3.0 \pm 0.1}$ at low temperatures below $T / T_{\mathrm{O}} \sim 0.5$, which is indicated by the broken line in the figure. On the other hand, $\operatorname{PrTi}_{2} \mathrm{Al}_{20}$ exhibit a convex curve in the full logarithmic plot, reflecting the gapped temperature dependence arising from the FQ ordering as discussed in the previous work[19]. Even though the temperature range for the power law behavior is somewhat narrow, the contrasting behavior between $\operatorname{PrV}_{2} \mathrm{Al}_{20}$ and $\operatorname{PrTi}_{2} \mathrm{Al}_{20}$ is evident. The power law behavior in $\mathrm{PrV}_{2} \mathrm{Al}_{20}$ suggests strong orbital fluctuation even in the multipolar ordered state. Interestingly, $\operatorname{PrTi}_{2} \mathrm{Al}_{20}$ under high pressure of $8.7 \mathrm{GPa}$ also indicates the same $T^{3}$ power law behavior[11]. Note that, for the sample with RRR 18 and 19, $\Delta \rho$ deviates upward from the power law behavior just below $T_{O}$, while it is not observed in the sample RRR with 5. This is supposed to reflect the fact that the multipolar transitions become sharper as RRR increases.

Next, we present the temperature dependence of the specific heat $(C)$ divided by $T$ in Figure. 2. Here, the lattice contribution is already subtracted by using $C / T$ for $\mathrm{LaV}_{2} \mathrm{Al}_{20}$. At the high temperatures, as we mentioned above, there are two peaks at $T_{\mathrm{Q}}=0.75 \mathrm{~K}$ and $T^{*}=0.65 \mathrm{~K}$ corresponding to the multipole orderings. Note that these temperatures are designated following the previous work[17] and we do not intend to refer to 
any specific order parameter in this paper. In the low temperatures, the data exhibit a slight increase on cooling below $0.12 \mathrm{~K}$ and finally indicate an anomaly due to SC transition at $T=0.046 \mathrm{~K}$. The low $T$ upturn in $C / T$ becomes evident in the normal state stabilized under the magnetic field of $20 \mathrm{mT}$ and is found to follow $C / T \sim T^{2}$ down to the lowest $T$ of $30 \mathrm{mK}$. As already discussed in the previous work[16], this contribution is most likely arising from a hyperfine enhanced nuclear magnetism. The $4 f$-electron contribution of the specific heat divided by $T, C_{4 f} / T$ is obtained after subtracting $C_{n} / T \sim T^{-2}$ coming from the nuclear magnetism. The zero-field SC anomaly is clearly seen in $C_{4 f} / T$, which is shown in the figure by the open circles. This confirms the bulk character of the SC. The nearly constant $C_{4 f} / T$ in the normal state provides an estimate of the large electronic coefficient $\gamma \sim 0.9 \mathrm{~J} / \mathrm{molK}^{2}$, consistent with the effective mass $m^{*} / m_{0} \sim 140$ estimated from the $B_{\mathrm{c} 2}$ curve[16].

The SC jump in $C / T$ is evaluated using the temperature dependence of $\Delta C / T \equiv C_{4 f} / T-\gamma$, as is shown in the inset of Figure 2. The jump reaches $0.3 \mathrm{~J} / \mathrm{molK}^{2}$, which provides direct evidence of the heavy-fermion $\mathrm{SC}$. The ratio obtained from the jump, $\Delta C / \gamma T_{\mathrm{c}} \sim 0.3$ is much smaller than the BCS value of 1.43. As already discussed in the previous work[16], the above estimated jump can be further increased if the sample quality is improved, which is the one of the important future issues. In the inset of Figure 2, $\Delta C / T$ in weak magnetic fields are also shown. The SC anomaly is slightly suppressed at $B=0.5 \mathrm{mT}$ and is not observed at $B=20$ $\mathrm{mT}$. $T_{\mathrm{c}}$ at $B=0.5 \mathrm{mT}$ is roughly estimated to be 0.042 $\mathrm{K}$, which is consistent with the previously reported $B_{\mathrm{c} 2}$ curve[16].

Corresponding to the contrasting behavior in the resistivity below $T_{\mathrm{O}}$, the specific heat data also exhibit contrasting behavior in $\operatorname{PrTi}_{2} \mathrm{Al}_{20}$ and $\operatorname{PrV}_{2} \mathrm{Al}_{20}$ below the multipolar ordering temperatures. In order to demonstrate this, we present the full logarithmic plot of $C_{4 f}$ versus temperature for both $\operatorname{PrV}_{2} \mathrm{Al}_{20}$ and $\operatorname{PrTi}_{2} \mathrm{Al}_{20}$ in Figure 3(a). As clearly seen in the figure, $\operatorname{PrTi}_{2} \mathrm{Al}_{20}$ shows the exponential $T$ dependence with the convex curve below $T_{\mathrm{O}} \sim 2.0 \mathrm{~K}$, which is arising from an excitation gap expected for FQ ordering. On the other hand, $\operatorname{PrV}_{2} \mathrm{Al}_{20}$ exhibits a clear power law behavior in $\Delta C \equiv C_{4 f}-\gamma T$ with $\Delta C \propto T^{4}$ below $T^{*}[16,20]$ as shown by open circles. This power law behavior indicates the existence of a gapless mode corresponding to the so-called" orbiton" proposed for various transition metal based systems[21]. The gapless mode in the multipolar ordered state in $\operatorname{PrV}_{2} \mathrm{Al}_{20}$ would be the signature of strong orbital fluctuations arising from the proximity
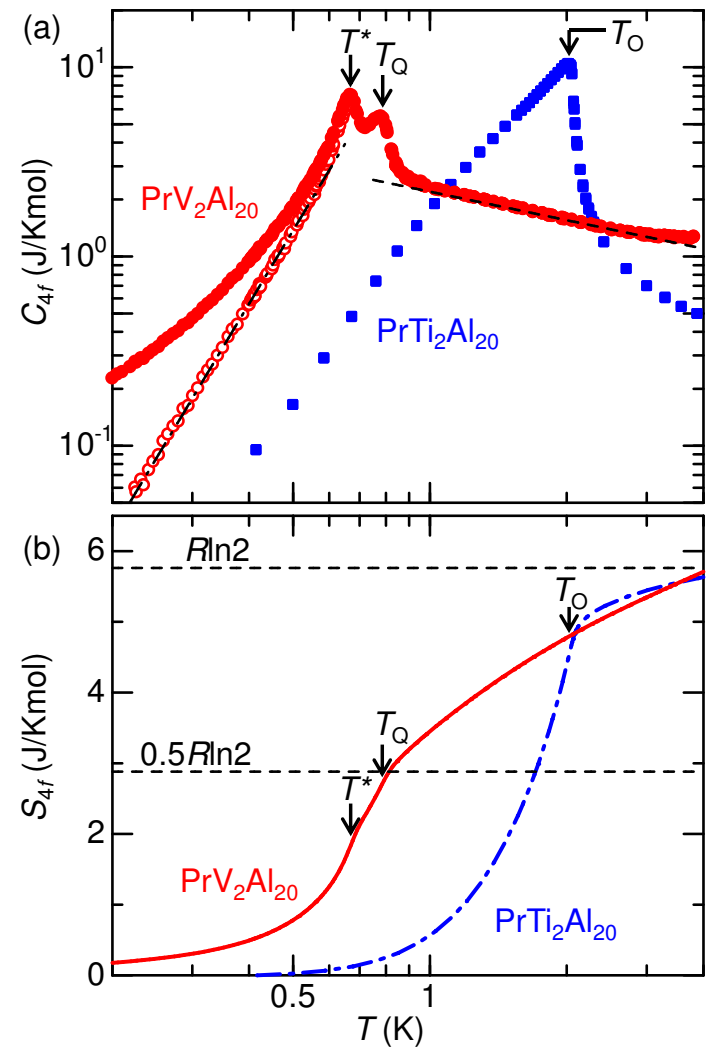

Figure 3: (a) Temperature dependence of $C_{4 f}$ for $\operatorname{PrV}_{2} \mathrm{Al}_{20}$ (closed circles) and $\operatorname{PrTi}_{2} \mathrm{Al}_{20}$ (closed squares) in full logarithmic scale. $\Delta C \equiv$ $C_{4 f}-\gamma T$ for $\operatorname{PrV}_{2} \mathrm{Al}_{20}$ is also shown by open circles. The dashed line below $T^{*}$ represents the $T^{4}$ behavior in $\Delta C$. The broken line above $T_{\mathrm{Q}}$ represents the $T^{-1 / 2}$ behavior in $C_{4 f}$. (b) Temperature dependence of the $4 f$ electron contribution to the entropy $S_{4 f}$ for $\operatorname{PrV}_{2} \mathrm{Al}_{20}$ (solid line) and $\operatorname{PrTi}_{2} \mathrm{Al}_{20}$ (dashed line). For details, see text.

to a orbital QCP. This gapless character should be the results of the strong $c$ - $f$ hybridization effect, which is also seen in the temperature dependence of $4 f$ electron contribution to the entropy $S_{4 f}$ obtained after the integration of $C_{4 f} / T$ vs $T$. As shown in Figure 3(b), while $S_{4 f}$ for $\mathrm{PrTi}_{2} \mathrm{Al}_{20}$ reaches $90 \%$ of $R \ln 2$ at $T_{\mathrm{O}} \sim 2.0 \mathrm{~K}, S_{4 f}$ for $\mathrm{PrV}_{2} \mathrm{Al}_{20}$ reaches only $50 \%$ of $R \ln 2$ at $T_{\mathrm{Q}} \sim 0.75 \mathrm{~K}$. Another $50 \%$ of $R \ln 2$ expected for $\Gamma_{3}$ doublet is already released at higher temperature range from $T_{\mathrm{Q}}$ to $T \sim 4$ $\mathrm{K}$. Interestingly, in the same temperature range, $C_{4 f}$ in $\operatorname{PrV}_{2} \mathrm{Al}_{20}$ exhibits the anomalous power law behavior of $C_{4 f} \propto T^{-1 / 2}$ (Fig. 3(a)), as already discussed in the previous work[16, 20]. In addition, in this temperature range, we have seen various anomalous metallic properties far different from the Fermi liquid behavior, such as the magnetic susceptibility proportional to $-T^{1 / 2}$ [10] and the resistivity proportional to $\ln T$ as indicated by 
the dashed line in Figure 1 (a). One possible scenario for the origin of the anomalous metal is the quadrupolar Kondo effect. Note that a residual entropy of $R / 2 \ln 2$ is expected for the quadrupolar Kondo effect, which is consistent with our observation of $S_{4 f}$ reaches only $50 \%$ of $R \ln 2$ in $\mathrm{PrV}_{2} \mathrm{Al}_{20}$ at $T_{\mathrm{Q}}$.

\section{Conclusion}

Here, we discussed the anomalous metallic behaviors found below and above the multipole ordering temperatures in a pure single crystal of $\operatorname{PrV}_{2} \mathrm{Al}_{20}$. We revealed that the resistivity of $\operatorname{PrV}_{2} \mathrm{Al}_{20}$ indicates power law temperature dependence proportional to $T^{3}$, which is in contrast to the gapped behavior found in $\operatorname{PrTi}_{2} \mathrm{Al}_{20}$. Likewise, the $4 f$ electron contribution to the specific heat of $\mathrm{PrV}_{2} \mathrm{Al}_{20}$ exhibits power law behavior proportional to $T^{4}$, which is again in contrast to the gapped behavior in $\operatorname{PrTi}_{2} \mathrm{Al}_{20}$. This suggests the existence of a gapless mode corresponding to" orbiton" in $\mathrm{PrV}_{2} \mathrm{Al}_{20}$. In addition, the $4 f$ electron contribution to the entropy in $\mathrm{PrV}_{2} \mathrm{Al}_{20}$ reaches only $50 \%$ of $R \ln 2$ at the orbital ordering at $T=0.75 \mathrm{~K}$, suggesting another $50 \%$ of $R \ln 2$ expected for $\Gamma_{3}$ doublet is already released at higher temperature possibly due to quadrupole Kondo effect. All these observations indicate the strong $c$ - $f$ hybridization and strong orbital fluctuations which may arise from the proximity to a orbital QCP in $\operatorname{PrV}_{2} \mathrm{Al}_{20}$. In order to clarify these possibilities, a lot of studies are required for both experiments and theories. In particular, the origin of the double transition found at $T_{\mathrm{Q}}=0.75$ and $T^{*}=0.65 \mathrm{~K}$ should be clarified through detailed studies of thermodynamics, transport and microscopic measurements. Further improvement of the sample quality is also the important future issue.

\section{Acknowledgments}

We thank Y. Uwatoko, K. Matsubayashi, J. Suzuki, T. Sakakibara, Y. Shimura, K. Araki, Y.-B. Kim, K. Kuga, $\mathrm{K}$. Hattori and K. Ueda for their support and the useful discussions. This Letter was partially supported by PRESTO of JST and Grants-in-Aid (Nos. 25707030 and 90422443) from the Japanese Society for the Promotion of Science (JSPS), by Grants-in-Aids for Scientific Research on Innovative Areas" Heavy Electrons " of the Ministry of Education, Culture, Sports, Science and Technology, Japan. The use of the facilities of the Materials Design and Characterization Laboratory at the Institute for Solid State Physics, The University of Tokyo is gratefully acknowledged. One of the authors,
(M. T.) was supported by the JSPS through Program for Leading Graduate Schools (MERIT).

\section{References}

[1] D. L. Cox, Quadrupolar kondo effect in uranium heavyelectron materials, Phys. Rev. Lett. 59 (11) (1987) 1240-1243. doi:10.1103/PhysRevLett.59.1240.

[2] G. R. Stewart, Non-fermi-liquid behavior in $d$ - and $f$ electron metals, Rev. Mod. Phys. 73 (2001) 797-855. doi:10.1103/RevModPhys.73.797.

[3] H. v. Löhneysen, A. Rosch, M. Vojta, P. Wölfle, Fermi-liquid instabilities at magnetic quantum phase transitions, Rev. Mod. Phys. 79 (2007) 1015-1075. doi:10.1103/RevModPhys.79.1015.

[4] P. Monthoux, D. Pines, G. G. Lonzarich, Superconductivity without phonons, Nature 450 (2007) 1177-1183.

[5] P. Morin, D. Schmitt, E. du Tremolet de Lacheisserie, Magnetic and quadrupolar properties of $\mathrm{PrPb}_{3}$, J. Magn. Magn. Mater. 30 (2) (1982) 257 - 264. doi:DOI: 10.1016/03048853(82)90206-2.

[6] H. Suzuki, M. Kasaya, T. Miyazaki, Y. Nemoto, T. Goto, Nonkramer's doublet ground state in prptbi, J. Phys. Soc. Jpn. 66 (9) (1997) 2566-2568. doi:10.1143/JPSJ.66.2566.

[7] T. Onimaru, T. Sakakibara, N. Aso, H. Yoshizawa, H. S. Suzuki, T. Takeuchi, Observation of modulated quadrupolar structures in $\mathrm{PrPb}_{3}$, Phys. Rev. Lett. 94 (19) (2005) 197201. doi:10.1103/PhysRevLett.94.197201.

[8] T. Onimaru, K. T. Matsumoto, Y. F. Inoue, K. Umeo, Y. Saiga, Y. Matsushita, R. Tamura, K. Nishimoto, I. Ishii, T. Suzuki, T. Takabatake, Superconductivity and structural phase transitions in caged compounds $R T_{2} \mathrm{Zn}_{20}(R=\mathrm{La}$, Pr, $T=\mathrm{Ru}$, Ir), J. Phys. Soc. Jpn. 79 (3) (2010) 033704. doi:10.1143/JPSJ.79.033704.

[9] T. Onimaru, K. T. Matsumoto, Y. F. Inoue, K. Umeo, T. Sakakibara, Y. Karaki, M. Kubota, T. Takabatake, Antiferroquadrupolar Ordering in a Pr-Based Superconductor $\operatorname{PrIr}_{2} \mathrm{Zn}_{20}$, Phys. Rev. Lett. 106 (17). doi:10.1103/PhysRevLett.106.177001.

[10] A. Sakai, S. Nakatsuji, Kondo effects and multipolar order in the cubic $\operatorname{PrTr}_{2} \mathrm{Al}_{20}(\mathrm{Tr}=\mathrm{Ti}, \mathrm{V})$, J. Phys. Soc. Jpn. 80 (6) (2011) 063701. doi:10.1143/JPSJ.80.063701.

[11] K. Matsubayashi, T. Tanaka, A. Sakai, S. Nakatsuji, Y. Kubo, Y. Uwatoko, Pressure-induced heavy fermion superconductivity in the nonmagnetic quadrupolar system prti2al20, Phys. Rev. Lett. 109 (2012) 187004. doi:10.1103/PhysRevLett.109.187004.

[12] T. J. Sato, S. Ibuka, Y. Nambu, T. Yamazaki, T. Hong, A. Sakai, S. Nakatsuji, Ferroquadrupolar ordering in $\operatorname{PrTi}_{2} \mathrm{Al}_{20}$, Phys. Rev. B 86 (2012) 184419. doi:10.1103/PhysRevB.86.184419.

[13] M. Koseki, Y. Nakanishi, K. Deto, G. Koseki, R. Kashiwazaki, F. Shichinomiya, M. Nakamura, M. Yoshizawa, A. Sakai, S. Nakatsuji, J. Phys. Soc. Jpn. 80 (2011) SA049.

[14] M. Matsunami, M. Taguchi, A. Chainani, R. Eguchi, M. Oura, A. Sakai, S. Nakatsuji, S. Shin, Kondo resonance in prti(2)al(20): Photoemission spectroscopy and single-impurity anderson model calculations, Phys. Rev. B 84 (19) (2011) 193101. doi:10.1103/PhysRevB.84.193101.

[15] Y. Tokunaga, H. Sakai, S. Kambe, A. Sakai, S. Nakatsuji, H. Harima, Phys. Rev. B 88 (2013) 085124. doi:10.1103/PhysRevB.88.085124.

[16] M. Tsujimoto, Y. Matsumoto, T. Tomita, A. Sakai, S. Nakatsuji, Heavy-fermion superconductivity in the quadrupole ordered state of $\operatorname{PrV}_{2} \mathrm{Al}_{20}$, Phys. Rev. Lett. 113 (2014) 267001. doi:10.1103/PhysRevLett.113.267001. 
[17] K. Araki, Y. Shimura, N. Kase, T. Sakakibara, A. Sakai, S. Nakatsuji, Magnetization and Specific Heat of the Cage Compound $\mathrm{PrV}_{2} \mathrm{Al}_{20}$, JPS Conf. Proc. 3 () (2014) 011093.

[18] T. U. Ito, W. Higemoto, K. Ninomiya, H. Luetkens, C. Baines, A. Sakai, S. Nakatsuji, $\mu$ SR Evidence of Nonmagnetic Order and ${ }^{141} \mathrm{Pr}$ Hyperfine-Enhanced Nuclear Magnetism in the Cubic $\Gamma_{3}$ Ground Doublet System $\mathrm{PrTi}_{2} \mathrm{Al}_{20}$, Journal of the Physical Society of Japan 80 (11) (2011) 113703. doi:10.1143/JPSJ.80.113703.

[19] A. Sakai, K. Kuga, S. Nakatsuji, Superconductivity in the ferroquadrupolar state in the quadrupolar kondo lattice $\operatorname{PrTi}_{2} \mathrm{Al}_{20}$, J. Phys. Soc. Jpn 81 (8) (2012) 083702. doi:10.1143/JPSJ.81.083702.

[20] M. Tsujimoto, Y. Matsumoto, S. Nakatsuji, Anomalous specific heat behaviour in the quadrupolar kondo system $\operatorname{PrV}_{2} \mathrm{Al}_{20}$, J. Phys. Conf.: Ser. 592 (2015) 01023. doi:10.1088/17426596/592/1/012023.

[21] S. Ishihara, Y. Murakami, T. Inami, K. Ishii, J. Mizuki, K. Hirota, S. Maekawa, Y. Endoh, Theory and experiment of orbital excitations in correlated oxides, New J. Phys. 7 (2005) 119. 